\title{
Spatial Estimates for the Constrained Anisotropic Elastic Cylinder
}

\author{
Stan Chiriţă • Michele Ciarletta
}

Received: 18 July 2006 / Accepted: 14 August 2006 /

Published online: 17 October 2006

(C) Springer Science + Business Media B.V. 2006

\begin{abstract}
This paper establishes spatial estimates in a prismatic (semi-infinite) cylinder occupied by an anisotropic homogeneous linear elastic material, whose elasticity tensor is strongly elliptic. The cylinder is maintained in equilibrium under zero body force, zero displacement on the lateral boundary and pointwise specified displacement over the base. The other plane end is subject to zero displacement (when the cylinder is finite, say). The limiting case of a semi-infinite cylinder is also considered and zero displacement on the remote end (at large distance) is not assumed in this case. A first approach is developed by considering two mean-square cross-sectional measures of the displacement vector whose spatial evolution with respect to the axial variable is studied by means of a technique based on a secondorder differential inequality. Conditions on the elastic constants are derived that show the cross-sectional measures exhibit alternative behaviour and in particular for the semi-infinite cylinder that there is either at least exponential growth or at most exponential decay. A second approach considers cross-sectional integrals involving the displacement and its gradient and furnishes information upon the spatial evolution, without restricting the range of strongly elliptic elastic constants. Such models are principally based upon a first-order differential inequality as well as on one of second order. The general results are explicitly presented for transversely isotropic materials and graphically illustrated for a cortical bone.
\end{abstract}

\footnotetext{
S. Chiriţă $(\bowtie)$

Faculty of Mathematics, Al. I. Cuza University of Iaşi, Blvd. Carol I, no. 11, 700506 Iaşi, Romania

e-mail: schirita@uaic.ro

M. Ciarletta

Dipartimento di Ingegneria dell'Informazione e Matematica Applicata, (DIIMA),

Università di Salerno, 84084 Fisciano SA, Italy

e-mail: ciarlett@diima.unisa.it
} 
Key words spatial estimates $\cdot$ anisotropic $\cdot$ strong elliptic elasticity tensor $\cdot$ linear elasticity $\cdot$ transverse isotropy $\cdot$ constrained cylinder

Mathematics Subject Classification (2000) 74B05

\section{Introduction}

We consider a prismatic cylinder occupied by an anisotropic homogeneous compressible linear elastic material having an elasticity tensor that is strongly elliptic. The cylinder is maintained in equilibrium under zero body force, zero displacement on the lateral boundary and pointwise specified displacement over the base. The other plane end is subject to zero displacement (when the cylinder is finite, say). The limiting case of a semi-infinite cylinder is also considered and zero displacement on the remote end (at large distance) is not assumed in this case.

The main purpose of this paper is to examine how certain cross-sectional and volume integral measures of the displacement vector evolve with respect to the axial variable, provided that the strong ellipticity of the elasticity tensor is assumed. To this end we develop two approaches based on the technique of first-order and secondorder differential inequalities.

A first approach is developed by considering two mean-square cross-sectional measures of the displacement vector whose spatial evolution with respect to the axial variable is studied by means of a technique based on a second-order differential inequality. Conditions on the elastic constants are derived that show the crosssectional measures exhibit alternative behaviour and in particular for the semiinfinite cylinder that there is either at least exponential growth or at most exponential decay.

The results are specialized for the isotropic materials and the dependence of the decay rate with respect to the parameter of the measure is studied and graphically illustrated for some well-known materials.

Furthermore, we are interested to find appropriate measures whose study can offer information on the spatial evolution of the displacement for the whole class of strongly elliptic anisotropic materials. To this end we develop a second approach which considers cross-sectional integrals involving the displacement and its gradient and furnishes information upon the spatial evolution, without restricting the range of strongly elliptic elastic coefficients. Such models are principally based upon a first-order differential inequality, but the technique of second-order differential inequality can also be used. The explicit results are presented for transversely isotropic materials and graphically illustrated for a cortical bone.

Our study in the present work was inspired by the fundamental paper of Flavin et al. [1] where such investigations were initiated for a constrained cylinder made of a homogeneous isotropic compressible linear elastic material. Two methods are developed there in order to study the spatial behaviour of the solution in a constrained elastic cylinder of variable cross section. The first method reduces the problem to a nonautonomous second-order differential inequality for the meansquare cross-sectional integral of the displacement, but it is valid only for a restricted range of the elastic moduli, which in terms of Poisson's ratio $v$ is characterized by the inequality $v<0.396$. The second approach, which relies upon a first-order 
differential inequality for a cross-sectional integral involving the displacement and its gradient, does not restrict the range of elastic moduli.

We have to recall that Biollay [2] has studied a similar problem for a semi-infinite isotropic elastic cylinder in which displacements are specified on the end and are required to vanish on the lateral surface. A spatial decay estimate is established for the cross-sectional mean square of the displacement vector and the decay rate depends only on Poisson's ratio and the smallest eigenvalue of the clamped membrane. Energy decay estimates have been established by Oleinik and Yosifian $[3,4]$ for certain problems involving the three-dimensional elasticity operator in noncylindrical regions in which the displacement is prescribed to vanish over part or all of the boundary. For the state of art on the Saint Venant principle we recommend the review papers by Horgan and Knowles [5] and Horgan [6, 7]. For a more recent review concerned with the effects of anisotropy, we refer to [8].

\section{Formulation of the Problem}

Let $\Omega$ denote a prismatic cylinder whose base, supposedly planar, is selected to lie in the $x_{1} O x_{2}$-coordinate plane of a three-dimensional rectangular Cartesian coordinate system and to contain the origin.

We suppose that the length of the cylinder is $L$ and that $D_{x_{3}} \subset \mathbb{R}^{2}$ represents the uniform bounded cross-section at distance $x_{3}$ from the origin. The boundary $\partial D$ of each cross-section is assumed sufficiently smooth to admit application of the divergence theorem in the plane of the cross-section. The cylinder consists of an anisotropic and homogeneous linear elastic material maintained in equilibrium under zero body-force. Therefore, the equilibrium equations yield

$$
C_{i j k l} u_{k, l j}=0, \quad x \in \Omega,
$$

where the components $C_{i j k l}$ of the elasticity tensor possess the major and minor symmetries

$$
C_{i j k l}=C_{k l i j}=C_{j i k l},
$$

and are assumed to be prescribed constant coefficients. The subscripts $i, j, k, l$ take values 1, 2, 3 and summation is implied by index repetition.

We recall that the elasticity tensor is positive definite if (Cf. Gurtin [9, p. 69,86])

$$
C_{i j k l} \phi_{i j} \phi_{k l}>0
$$

for every non-zero symmetric tensor $\phi_{i j}$. Further, $C_{i j k l}$ is strongly elliptic if

$$
C_{i j k l} \xi_{i} \xi_{k} \eta_{j} \eta_{l}>0
$$

for every non-zero vectors $\xi_{i}$ and $\eta_{j}$.

For an isotropic elastic material the components of the elasticity tensor are given by

$$
C_{i j k l}=\lambda \delta_{i j} \delta_{k l}+\mu\left(\delta_{i k} \delta_{j l}+\delta_{i l} \delta_{j k}\right),
$$


where $\lambda$ and $\mu$ are the Lamé moduli. In this case the elasticity tensor is positive definite if and only if the Lamé moduli obey the inequalities

$$
\mu>0, \quad 3 \lambda+2 \mu>0
$$

while the elasticity tensor is strongly elliptic if and only if

$$
\mu>0, \quad \lambda+2 \mu>0 .
$$

Throughout the paper, zero body force is assumed, and it is supposed that the cylinder is in equilibrium under prescribed displacement on the end $x_{3}=0$ and zero displacement on the lateral surface. The other plane end $x_{3}=L$ is subject to zero displacement (when $L$ is finite, say). The limiting case $L \rightarrow \infty$ is also considered and zero displacement on the remote end (at large distances) is not assumed in this case.

We assume the existence of a sufficiently smooth displacement vector $u_{i}(x)$ satisfying the equilibrium Eq. (2.1) and the following boundary conditions of our problem:

$$
\begin{aligned}
& u_{i}(x)=0 \text { for } x \in \partial D_{x_{3}}, x_{3} \in[0, L], \\
& u_{i}(x)=g_{i}(x), \quad x \in D_{0}, \\
& u_{i}(x)=0, \quad x \in D_{L},
\end{aligned}
$$

in the case where $L$ is finite (say). In the limiting case $L \rightarrow \infty$ a condition of the type (2.10) is unnecessary. In the above relations, $g_{i}\left(x_{1}, x_{2}\right)$ are specified functions.

\section{Some Displacement Cross-Sectional Measures}

Throughout this paper we will consider the class of elastic materials whose elasticity tensor is strongly elliptic. We will specify some subclasses of elastic materials for which it is possible to study the spatial behaviour by using an appropriate displacement cross-sectional measure. In fact, we will discuss spatial decay estimates for the displacement vector measured by an $L_{2}$-norm over a plane cross-section of the cylinder. To this end we note that the strong ellipticity condition (2.4) implies that

$$
C_{i 3 k 3} \xi_{i} \xi_{k}>0
$$

for all non-zero vectors $\xi_{i}$ and

$$
C_{i \alpha k \beta} \xi_{i} \xi_{k} \eta_{\alpha} \eta_{\beta}>0
$$

for all non-zero vectors $\xi_{i}$ and $\eta_{\alpha}$. The Greek subscripts $\alpha$ and $\beta$ take values 1,2 and summation is implied by index repetition. In what follows we will denote by $\varkappa_{m}$ and $\varkappa_{M}$ the minimum and maximum eigenvalues of the tensor $C_{i 3 k 3}$ so that we have

$$
\varkappa_{m} \xi_{i} \xi_{i} \leq C_{i 3 k 3} \xi_{i} \xi_{k} \leq \varkappa_{M} \xi_{i} \xi_{i}
$$


Moreover, in view of the assumption (3.2) and by using the Gärding inequality (cf. Fichera [10, p. 366]), it follows that there exists a constant $\gamma_{0}>0$ such that

$$
\int_{D_{x_{3}}} C_{i \alpha k \beta} v_{i, \alpha} v_{k, \beta} \mathrm{d} a \geq \gamma_{0} \int_{D_{x_{3}}} v_{i, \alpha} v_{i, \alpha} \mathrm{d} a,
$$

for every vector field $v_{i}$ satisfying the boundary condition (2.8).

\subsection{Monoclinic Materials with $x_{1} O x_{2}$ as a Plane Elastic Symmetry}

In this subsection we consider elastic materials with

$$
C_{\alpha 333}=0, \quad C_{3 \alpha \beta \gamma}=0,
$$

which include the monoclinic systems (with 13 elasticities) having $x_{1} O x_{2}$ as a plane of elastic symmetry, the rhombic system (with nine elasticities), the tetragonal system (with six and seven elasticities), the cubic system (with three elasticities) and the hexagonal systems (with five and six elasticities), (cf. Gurtin [9, p. 87]). In order to study the spatial behaviour of the solution we introduce the following displacement cross-sectional measure

$$
I\left(x_{3}\right)=\int_{D_{x_{3}}}\left(C_{\alpha 3 \beta 3} u_{\alpha} u_{\beta}+\delta C_{3333} u_{3}^{2}\right) \mathrm{d} a, \quad x_{3} \geq 0,
$$

with $\delta$ a strictly positive parameter at our disposal. This is, indeed, an acceptable cross-sectional measure as the integrand is positive definite in $u_{i}$, in view of Eq. (3.1).

By direct differentiation we obtain

$$
I^{\prime}\left(x_{3}\right)=2 \int_{D_{x_{3}}}\left(C_{\alpha 3 \beta 3} u_{\alpha} u_{\beta, 3}+\delta C_{3333} u_{3} u_{3,3}\right) \mathrm{d} a
$$

and

$$
\begin{aligned}
I^{\prime \prime}\left(x_{3}\right)= & 2 \int_{D_{x_{3}}}\left(C_{\alpha 3 \beta 3} u_{\alpha, 3} u_{\beta, 3}+\delta C_{3333} u_{3,3}^{2}\right) \mathrm{d} a \\
& +2 \int_{D_{x_{3}}}\left(C_{\alpha 3 \beta 3} u_{\alpha} u_{\beta, 33}+\delta C_{3333} u_{3} u_{3,33}\right) \mathrm{d} a .
\end{aligned}
$$

Now, with the assumption (3.5), from the equilibrium Eq. (2.1) we deduce that

$$
\begin{aligned}
& C_{\alpha 3 \beta 3} u_{\beta, 33}=-\left(C_{\alpha \beta \mu \lambda} u_{\mu, \lambda}\right)_{, \beta}-\left(C_{\alpha \beta 33} u_{3,3}\right)_{, \beta}-C_{\alpha 33 \lambda} u_{3,3 \lambda}, \\
& C_{3333} u_{3,33}=-\left(C_{3 \alpha 3 \beta} u_{3, \beta}\right)_{, \alpha}-\left(C_{3 \alpha 3 \lambda} u_{\lambda, 3}\right)_{, \alpha}-C_{33 \beta \alpha} u_{\beta, 3 \alpha} .
\end{aligned}
$$


If we substitute the relations (3.9) and (3.10) into Eq. (3.8) and then we use integration by parts and the boundary condition (2.8), we deduce that

$$
\begin{aligned}
I^{\prime \prime}\left(x_{3}\right)=2 \int_{D_{x_{3}}}\left[\delta C_{3333} u_{3,3}^{2}+\left(C_{\alpha \beta 33}+C_{3 \alpha 3 \beta}\right) u_{3,3} u_{\alpha, \beta}\right. \\
\left.\quad+C_{\alpha \beta \lambda \mu} u_{\alpha, \beta} u_{\lambda, \mu}\right] \mathrm{d} a+2 \int_{D_{x_{3}}}\left[C_{\alpha 3 \beta 3} u_{\alpha, 3} u_{\beta, 3}\right. \\
\quad+\delta\left(C_{3 \alpha 3 \beta}+C_{33 \alpha \beta}\right) u_{3, \alpha} u_{\beta, 3} \\
\left.+\delta C_{\alpha 3 \beta 3} u_{3, \alpha} u_{3, \beta}\right] \mathrm{d} a .
\end{aligned}
$$

Further, in view of the assumption (3.2) and the boundary condition (2.8), by the Gärding inequality (Cf. Fichera [10, p. 366]) it follows that there exists a constant $\gamma_{0}>0$ such that

$$
\int_{D_{x_{3}}} C_{\alpha \beta \lambda \mu} u_{\alpha, \beta} u_{\lambda, \mu} \mathrm{d} a \geq \gamma_{0} \int_{D_{x_{3}}} u_{\alpha, \beta} u_{\alpha, \beta} \mathrm{d} a .
$$

Therefore, by choosing the parameter $\delta$ to satisfy

$$
\delta>\frac{\left(C_{\alpha \beta 33}+C_{3 \alpha 3 \beta}\right)\left(C_{\alpha \beta 33}+C_{3 \alpha 3 \beta}\right)}{4 C_{3333} \gamma_{0}}
$$

we can determine the constant $c_{1}>0$ so that

$$
\begin{aligned}
2 \int_{D_{x_{3}}} & {\left[\delta C_{3333} u_{3,3}^{2}+\left(C_{\alpha \beta 33}+C_{3 \alpha 3 \beta}\right) u_{3,3} u_{\alpha, \beta}\right.} \\
& \left.+C_{\alpha \beta \lambda \mu} u_{\alpha, \beta} u_{\lambda, \mu}\right] \mathrm{d} a \geq c_{1} \int_{D_{x_{3}}} u_{\alpha, \beta} u_{\alpha, \beta} \mathrm{d} a .
\end{aligned}
$$

Moreover, we choose the parameter $\delta$ to satisfy

$$
0<\delta<\frac{4 \hat{\varkappa}_{m}^{2}}{\left(C_{\alpha \beta 33}+C_{3 \alpha 3 \beta}\right)\left(C_{\alpha \beta 33}+C_{3 \alpha 3 \beta}\right)},
$$

where $\hat{\varkappa}_{m}$ is the minimum eigenvalue of the tensor $C_{\alpha 3 \beta 3}$. Then, we can determine the constant $c_{2}>0$ so that

$$
\begin{array}{r}
2 \int_{D_{x_{3}}}\left[C_{\alpha 3 \beta 3} u_{\alpha, 3} u_{\beta, 3}+\delta\left(C_{3 \alpha 3 \beta}+C_{33 \alpha \beta}\right) u_{3, \alpha} u_{\beta, 3}\right. \\
\left.+\delta C_{\alpha 3 \beta 3} u_{3, \alpha} u_{3, \beta}\right] \mathrm{d} a \geq c_{2} \int_{D_{x_{3}}} u_{3, \alpha} u_{3, \alpha} \mathrm{d} a .
\end{array}
$$

Concluding, we can see that, at least in the class of strongly elliptic materials characterized by the inequality

$$
\left(C_{\alpha \beta 33}+C_{3 \alpha 3 \beta}\right)\left(C_{\alpha \beta 33}+C_{3 \alpha 3 \beta}\right)<4 \hat{\varkappa}_{m} \sqrt{\gamma_{0} C_{3333}},
$$


the choices (3.13) and (3.15) are simultaneously possible and hence with $\delta$ satisfying

$$
\frac{\left(C_{\alpha \beta 33}+C_{3 \alpha 3 \beta}\right)\left(C_{\alpha \beta 33}+C_{3 \alpha 3 \beta}\right)}{4 C_{3333} \gamma_{0}}<\delta<\frac{4 \hat{\varkappa}_{m}^{2}}{\left(C_{\alpha \beta 33}+C_{3 \alpha 3 \beta}\right)\left(C_{\alpha \beta 33}+C_{3 \alpha 3 \beta}\right)},
$$

the relations (3.11), (3.14) and (3.16) furnish

$$
I^{\prime \prime}\left(x_{3}\right) \geq c_{1} \int_{D_{x_{3}}} u_{\alpha, \beta} u_{\alpha, \beta} \mathrm{d} a+c_{2} \int_{D_{x_{3}}} u_{3, \alpha} u_{3, \alpha} \mathrm{d} a .
$$

In what follows, we shall assume that the constants $\delta, c_{1}$ and $c_{2}$ are chosen so that the inequalities (3.14) and (3.16) hold true and therefore, the estimate (3.19) is true.

On the other hand, by using the Cauchy-Schwarz inequality, from Eq. (3.6) we deduce that

$$
I\left(x_{3}\right) \leq \hat{\varkappa}_{M} \int_{D_{x_{3}}} u_{\alpha} u_{\alpha} \mathrm{d} a+\delta C_{3333} \int_{D_{x_{3}}} u_{3}^{2} \mathrm{~d} a,
$$

where $\hat{\varkappa}_{M}$ is the maximum eigenvalue of the tensor $C_{\alpha 3 \beta 3}$. Further, the boundary condition (2.8) allows us to write the following inequalities:

$$
\begin{gathered}
\lambda_{0} \int_{D_{x_{3}}} u_{\alpha} u_{\alpha} \mathrm{d} a \leq \int_{D_{x_{3}}} u_{\alpha, \beta} u_{\alpha, \beta} \mathrm{d} a, \\
\lambda_{0} \int_{D_{x_{3}}} u_{3}^{2} \mathrm{~d} a \leq \int_{D_{x_{3}}} u_{3, \beta} u_{3, \beta} \mathrm{d} a,
\end{gathered}
$$

where $\lambda_{0}>0$ is the first eigenvalue in the two-dimensional clamped membrane eigenvalue problem for the cross section $D_{x_{3}}$. Thus, by combining the relations (3.20) to (3.22) we deduce that

$$
I\left(x_{3}\right) \leq \hat{\varkappa}_{M} \lambda_{0}^{-1} \int_{D_{x_{3}}} u_{\alpha, \beta} u_{\alpha, \beta} \mathrm{d} a+\delta C_{3333} \lambda_{0}^{-1} \int_{D_{x_{3}}} u_{3, \beta} u_{3, \beta} \mathrm{d} a .
$$

Moreover, from the relations (3.19) and (3.23) we deduce the following second-order differential inequality

$$
I^{\prime \prime}\left(x_{3}\right)-k_{1}^{2} I\left(x_{3}\right) \geq 0 \text { for all } x_{3} \geq 0,
$$

with

$$
k_{1}=\sqrt{\lambda_{0} \min \left\{\frac{c_{1}}{\hat{\varkappa}_{M}}, \frac{c_{2}}{\delta C_{3333}}\right\}} .
$$

We now proceed to integrate the differential inequality (3.24). To this end we use a well-known Comparison Principle (a generalisation of the curve under chord property for convex functions; e.g., [11, p. 124]). On this basis we conclude that $I\left(x_{3}\right)$ is bounded above by $H\left(x_{3}\right)$, the solution of the differential equation corresponding to the differential inequality (3.24) with the same boundary conditions, that is

$$
H^{\prime \prime}\left(x_{3}\right)-k_{1}^{2} H\left(x_{3}\right)=0 \text { for all } x_{3} \in[0, L],
$$

and

$$
H(0)=I(0), \quad H(L)=I(L) .
$$


This yields

$$
\begin{aligned}
I\left(x_{3}\right) & \leq \frac{1-e^{-2 k_{1}\left(L-x_{3}\right)}}{1-e^{-2 k_{1} L}} I(0) e^{-k_{1} x_{3}}+\frac{1-e^{-2 k_{1} x_{3}}}{1-e^{-2 k_{1} L}} I(L) e^{-k_{1}\left(L-x_{3}\right)} \leq \\
& \leq I(0) e^{-k_{1} x_{3}}+I(L) e^{-k_{1}\left(L-x_{3}\right)} \quad \text { for all } x_{3} \in[0, L] .
\end{aligned}
$$

The following result is a direct consequence of the estimate (3.28).

Theorem 1 In the context of the finite cylinder consisting of homogeneous monoclinic material and subject to the end condition (2.10), or for the semi-infinite cylinder, for which $L \rightarrow \infty$, with

$$
\lim _{L \rightarrow \infty} I(L) e^{-k_{1} L}=0,
$$

the cross-sectional measure (3.6) satisfies the decay law

$$
I\left(x_{3}\right) \leq I(0) e^{-k_{1} x_{3}}, \quad x_{3} \geq 0,
$$

where $k_{1}$ is given by Eq. (3.25).

Remark 1 The decay estimate (3.30) may be made fully explicit by expressing $I(0)$ in terms of the given data. In fact, if we substitute the end condition (2.9) into relation (3.6), then we have

$$
I(0)=\int_{D_{0}}\left(C_{\alpha 3 \beta 3} g_{\alpha} g_{\beta}+\delta C_{3333} g_{3}^{2}\right) \mathrm{d} a .
$$

It is instructive to specialize our results to the case of an isotropic material. Then $\hat{\varkappa}_{m}=\mu, \hat{\varkappa}_{M}=\mu, C_{3333}=\lambda+2 \mu, \gamma_{0}=\min (\mu, 2 \lambda+3 \mu)$ for $\mu>0, \lambda+\frac{2}{3} \mu>0$ and $\gamma_{0}=\min (\lambda+2 \mu,-\lambda)$ for $\lambda<0, \lambda+2 \mu>0$. Moreover, by means of the arithmeticgeometric mean inequality we obtain

$$
\begin{aligned}
2 \int_{D_{x_{3}}} & {\left[\delta(\lambda+2 \mu) u_{3,3}^{2}+\mu u_{\alpha, \beta} u_{\alpha, \beta}+(\lambda+\mu) u_{3,3} u_{\varrho, \varrho}+(\lambda+\mu) u_{\alpha, \alpha} u_{\varrho, \varrho}\right] \mathrm{d} a \geq } \\
\geq & {\left[2 \delta(\lambda+2 \mu)-|\lambda+\mu| \varepsilon_{1}\right] \int_{D_{x_{3}}} u_{3,3}^{2} \mathrm{~d} a+\left(2 \mu-\varepsilon_{2}\right) \int_{D_{x_{3}}} u_{\alpha, \beta} u_{\alpha, \beta} \mathrm{d} a+} \\
& +\int_{D_{x_{3}}}\left\{\varepsilon_{2} u_{\alpha, \beta} u_{\alpha, \beta}+\left[2(\lambda+\mu)-\frac{1}{\varepsilon_{1}}|\lambda+\mu|\right] u_{\alpha, \alpha} u_{\varrho, \varrho}\right\} \mathrm{d} a,
\end{aligned}
$$

where $\varepsilon_{1}>0$ and $\varepsilon_{2}$ are constants at our disposal. If we use the inequality (as in the paper by Flavin et al. [1])

$$
\int_{D_{x_{3}}} u_{\alpha, \beta} u_{\alpha, \beta} \mathrm{d} a \geq \int_{D_{x_{3}}} u_{\alpha, \alpha} u_{\varrho, \varrho} \mathrm{d} a,
$$


then Eq. (3.32) yields

$$
\begin{aligned}
2 \int_{D_{x_{3}}} & {\left[\delta(\lambda+2 \mu) u_{3,3}^{2}+\mu u_{\alpha, \beta} u_{\alpha, \beta}+(\lambda+\mu) u_{3,3} u_{\varrho, \varrho}+(\lambda+\mu) u_{\alpha, \alpha} u_{\varrho, \varrho}\right] \mathrm{d} a \geq } \\
\geq & {\left[2 \delta(\lambda+2 \mu)-|\lambda+\mu| \varepsilon_{1}\right] \int_{D_{x_{3}}} u_{3,3}^{2} \mathrm{~d} a+\left(2 \mu-\varepsilon_{2}\right) \int_{D_{x_{3}}} u_{\alpha, \beta} u_{\alpha, \beta} \mathrm{d} a+} \\
+ & {\left[\varepsilon_{2}+2(\lambda+\mu)-\frac{1}{\varepsilon_{1}}|\lambda+\mu|\right] \int_{D_{x_{3}}} u_{\alpha, \alpha} u_{\varrho, \varrho} \mathrm{d} a . }
\end{aligned}
$$

Thus, if we set

$$
\varepsilon_{1}=\frac{2 \delta(\lambda+2 \mu)}{|\lambda+\mu|}, \quad \varepsilon_{2}=\frac{2(\lambda+\mu)}{\delta}\left[-\delta+\frac{\lambda+\mu}{4(\lambda+2 \mu)}\right],
$$

then the relation (3.14) will hold with

$$
c_{1}=2 \mu-\varepsilon_{2}=2(\lambda+2 \mu)-\frac{(\lambda+\mu)^{2}}{2 \delta(\lambda+2 \mu)}>0,
$$

provided

$$
\delta>\frac{(\lambda+\mu)^{2}}{4(\lambda+2 \mu)^{2}} .
$$

Furthermore, we have

$$
\begin{aligned}
2 \int_{D_{x_{3}}} & {\left[\mu u_{\alpha, 3} u_{\alpha, 3}+\delta(\lambda+\mu) u_{\alpha, 3} u_{3, \alpha}+\delta \mu u_{3, \alpha} u_{3, \alpha}\right] \mathrm{d} a \geq } \\
\geq & {\left[2 \mu-\delta|\lambda+\mu| \varepsilon_{3}\right] \int_{D_{x_{3}}} u_{\alpha, 3} u_{\alpha, 3} \mathrm{~d} a } \\
+ & {\left[2 \delta \mu-\frac{\delta|\lambda+\mu|}{\varepsilon_{3}}\right] \int_{D_{x_{3}}} u_{3, \alpha} u_{3, \alpha} \mathrm{d} a, }
\end{aligned}
$$

with $\varepsilon_{3}$ a strictly positive constant at our disposal. If we set

$$
\varepsilon_{3}=\frac{2 \mu}{\delta|\lambda+\mu|},
$$

then the relation (3.16) will hold with

$$
c_{2}=2 \delta \mu-\frac{\delta|\lambda+\mu|}{\varepsilon_{3}}=2 \delta \mu-\frac{\delta^{2}|\lambda+\mu|^{2}}{2 \mu}>0,
$$

provided

$$
\delta<\frac{4 \mu^{2}}{(\lambda+\mu)^{2}}
$$


Figure 1 Dependence of the decay rate $k_{1}$ on the parameter $\delta$.

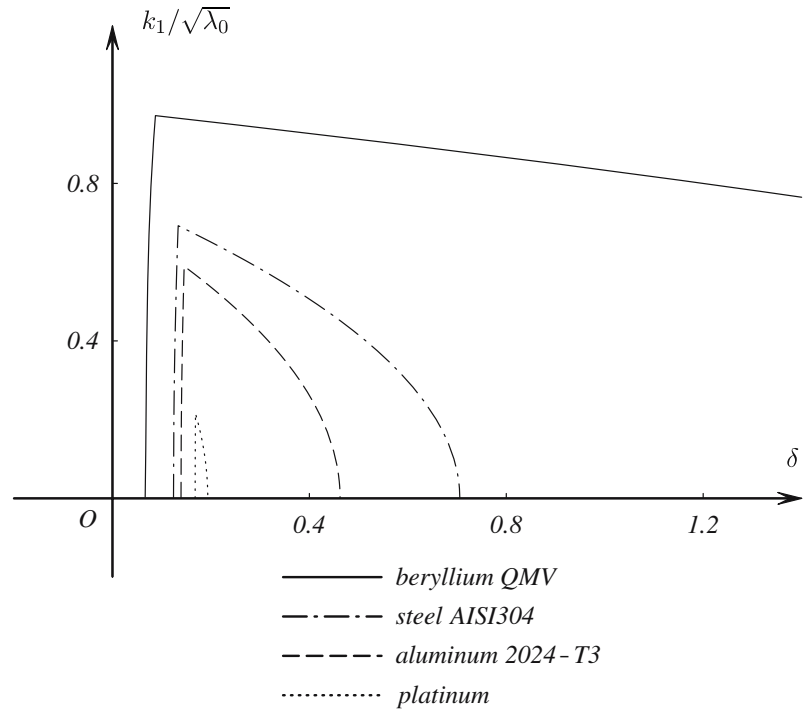

Concluding, the estimate (3.30) holds true with

$$
k_{1}=\sqrt{\frac{\lambda_{0}}{2 \delta \mu(\lambda+2 \mu)} \min \left\{4 \delta(\lambda+2 \mu)^{2}-(\lambda+\mu)^{2}, 4 \delta \mu^{2}-\delta^{2}(\lambda+\mu)^{2}\right\}},
$$

in the class of elastic materials characterized by

$$
\frac{(\lambda+\mu)^{2}}{4(\lambda+2 \mu)^{2}}<\frac{4 \mu^{2}}{(\lambda+\mu)^{2}},
$$

and with $\delta$ taking any value in the interval

$$
\left(\frac{(\lambda+\mu)^{2}}{4(\lambda+2 \mu)^{2}}, \frac{4 \mu^{2}}{(\lambda+\mu)^{2}}\right) .
$$

It is worth pointing out that the relation (3.43) characterizes the class of elastic materials considered in [1] (that is the class of elastic materials with the Poisson's ratio $v<0.396)$.

The dependence of the decay rate $k_{1}$ with respect to the parameter $\delta$ is graphically illustrated in Figure 1 for platinum (with the Poisson ratio $v=0.39$ ), alluminum 2024$T 3$ (with $v=0.33)$, steel AISI $304(v=0.29)$ and beryllium $Q M V(v=0.03)$.

\subsection{General Anisotropic Materials}

In this subsection we consider the class of anisotropic materials referred to as triclinic materials (cf. Gurtin [9]). Essentially in this subsection we consider the case when

$$
C_{\alpha 333} \neq 0 \text { or } C_{\alpha \beta \gamma 3} \neq 0 .
$$

If we set

$$
m^{2}=\left(C_{i \alpha k 3}+C_{i 3 k \alpha}\right)\left(C_{i \alpha k 3}+C_{i 3 k \alpha}\right),
$$


then, by means of the arithmetic-geometric mean inequality and the Schwarz inequality, we get

$$
\begin{gathered}
\int_{D_{x_{3}}}\left[C_{i 3 k 3} v_{i, 3} v_{k, 3}+C_{i \alpha k \beta} v_{i, \alpha} v_{k, \beta}+\left(C_{i \alpha k 3}+C_{i 3 k \alpha}\right) v_{i, \alpha} v_{k, 3}\right] \mathrm{d} a \geq \\
\geq \int_{D_{x_{3}}}\left[\left(\varkappa_{m}-\frac{\varepsilon_{4}}{2} m\right) v_{i, 3} v_{i, 3}+\left(\gamma_{0}-\frac{1}{2 \varepsilon_{4}} m\right) v_{i, \alpha} v_{i, \alpha}\right] \mathrm{d} a,
\end{gathered}
$$

for every strictly positive constant $\varepsilon_{4}$ at our disposal. Thus, if we set $\varepsilon_{4} m=2 \varkappa_{m}$ then we get

$$
\int_{D_{x_{3}}}\left[C_{i 3 k 3} v_{i, 3} v_{k, 3}+C_{i \alpha k \beta} v_{i, \alpha} v_{k, \beta}+\left(C_{i \alpha k 3}+C_{i 3 k \alpha}\right) v_{i, \alpha} v_{k, 3}\right] \mathrm{d} a \geq C_{0} \int_{D_{x_{3}}} v_{i, \alpha} v_{i, \alpha} \mathrm{d} a,
$$

where

$$
C_{0}=\gamma_{0}-\frac{m^{2}}{4 \varkappa_{m}}>0,
$$

provided the constitutive restriction

$$
m^{2}<4 \gamma_{0} \varkappa_{m}
$$

is assumed. On this basis we will assume that there is a positive constant $c_{0}$ such that

$$
\int_{D_{x_{3}}}\left[C_{i 3 k 3} v_{i, 3} v_{k, 3}+C_{i \alpha k \beta} v_{i, \alpha} v_{k, \beta}+\left(C_{i \alpha k 3}+C_{i 3 k \alpha}\right) v_{i, \alpha} v_{k, 3}\right] \mathrm{d} a \geq c_{0} \int_{D_{x_{3}}} v_{i, \alpha} v_{i, \alpha} \mathrm{d} a,
$$

for every vector field $v_{i}$ satisfying a homogeneous boundary condition of type described by the relation (2.8).

On this basis we will consider the function $J\left(x_{3}\right)$ defined by

$$
J\left(x_{3}\right)=\int_{D_{x_{3}}} C_{i 3 k 3} u_{i} u_{k} \mathrm{~d} a,
$$

which is an acceptable measure because the integrand is positive definite in $u_{i}$. We derive a second-order differential inequality for Eq. (3.52) and derive the evolution properties of the measure by integration of the inequality.

Successive differentiation of Eq. (3.52) gives

$$
J^{\prime}\left(x_{3}\right)=2 \int_{D_{x_{3}}} C_{i 3 k 3} u_{i} u_{k, 3} \mathrm{~d} a,
$$

and

$$
J^{\prime \prime}\left(x_{3}\right)=2 \int_{D_{x_{3}}} C_{i 3 k 3} u_{i, 3} u_{k, 3} \mathrm{~d} a+2 \int_{D_{x_{3}}} C_{i 3 k 3} u_{i} u_{k, 33} \mathrm{~d} a .
$$

Further, the equilibrium Eq. (2.1) gives

$$
C_{i 3 k 3} u_{k, 33}=-\left(C_{i \alpha k \beta} u_{k, \beta}\right)_{, \alpha}-\left(C_{i \alpha k 3} u_{k, 3}\right)_{, \alpha}-C_{i 3 k \beta} u_{k, 3 \beta},
$$


so that the relation (3.54) becomes

$$
\begin{aligned}
J^{\prime \prime}\left(x_{3}\right)= & 2 \int_{D_{x_{3}}} C_{i 3 k 3} u_{i, 3} u_{k, 3} \mathrm{~d} a \\
& -2 \int_{D_{x_{3}}} u_{i}\left[\left(C_{i \alpha k \beta} u_{k, \beta}\right)_{, \alpha}+\left(C_{i \alpha k 3} u_{k, 3}\right)_{, \alpha}+C_{i 3 k \beta} u_{k, 3 \beta}\right] \mathrm{d} a .
\end{aligned}
$$

By using an integration by parts and the lateral boundary conditions (2.8), from (3.56) we get

$$
J^{\prime \prime}\left(x_{3}\right)=2 \int_{D_{x_{3}}}\left[C_{i 3 k 3} u_{i, 3} u_{k, 3}+C_{i \alpha k \beta} u_{i, \alpha} u_{k, \beta}+\left(C_{i \alpha k 3}+C_{i 3 k \alpha}\right) u_{i, \alpha} u_{k, 3}\right] \mathrm{d} a
$$

and hence, by means of the relation (3.51), we obtain

$$
J^{\prime \prime}\left(x_{3}\right) \geq 2 c_{0} \int_{D_{x_{3}}} u_{i, \alpha} u_{i, \alpha} \mathrm{d} a .
$$

On the other hand, by using the relation (3.3), from (3.52) we deduce that

$$
J\left(x_{3}\right) \leq \varkappa_{M} \int_{D_{x_{3}}} u_{i} u_{i} \mathrm{~d} a .
$$

Thus, by combining the relations (3.21), (3.22) and (3.59) we deduce that

$$
J\left(x_{3}\right) \leq \varkappa_{M} \lambda_{0}^{-1} \int_{D_{x_{3}}} u_{i, \alpha} u_{i, \alpha} \mathrm{d} a .
$$

Moreover, from the relations (3.58) and (3.60), we deduce the following second-order differential inequality

$$
J^{\prime \prime}\left(x_{3}\right)-k_{2}^{2} J\left(x_{3}\right) \geq 0 \text { for all } x_{3} \geq 0,
$$

with

$$
k_{2}=\sqrt{\frac{2 c_{0} \lambda_{0}}{\varkappa_{M}}}
$$

The differential inequality (3.61) gives spatial estimates of the general type (3.30) for the spatial behaviour of the displacement in the class of anisotropic elastic materials for which (3.51) holds true.

\section{Some Measures Involving the Displacement and its First Gradient}

Throughout this section we will consider the most general anisotropic materials satisfying the strong ellipticity condition. Unlike the approach considered in the previous section, the argument used, while in passing involves volume integrals of the displacement and its first gradient, do not further restrict the elastic constants. 


\subsection{First Measure}

Let us introduce the cross-sectional function

$$
\mathcal{K}_{1}\left(x_{3}\right)=-\int_{D_{x_{3}}} \tau_{3 i} u_{i} \mathrm{~d} a, \quad x_{3} \in[0, L],
$$

with $\tau_{i j}$ the stress components, that is

$$
\tau_{i j}=C_{i j k l} u_{k, l}
$$

Thus, we have

$$
\mathcal{K}_{1}\left(x_{3}\right)=-\int_{D_{x_{3}}} C_{i 3 k l} u_{k, l} u_{i} \mathrm{~d} a, \quad x_{3} \in[0, L],
$$

and hence, by direct differentiation, we obtain

$$
\mathcal{K}_{1}^{\prime}\left(x_{3}\right)=-\int_{D_{x_{3}}}\left(C_{i 3 k l} u_{k, l 3} u_{i}+C_{i 3 k l} u_{k, l} u_{i, 3}\right) \mathrm{d} a, \quad x_{3} \in[0, L] .
$$

By using the equilibrium Eq. (3.55) and by an integration by parts with the use of the lateral boundary condition (2.8), we deduce that

$$
\mathcal{K}_{1}^{\prime}\left(x_{3}\right)=-\int_{D_{x_{3}}} C_{i j k l} u_{i, j} u_{k, l} \mathrm{~d} a .
$$

For later convenience, we integrate the relation (4.5) with respect to $x_{3}$ over the interval $\left[x_{3}, L\right]$, to obtain

$$
\mathcal{K}_{1}(L)-\mathcal{K}_{1}\left(x_{3}\right)=-\int_{\Omega_{x_{3}}} C_{i j k l} u_{i, j} u_{k, l} \mathrm{~d} v
$$

where $\Omega_{x_{3}}=\Omega \backslash\left\{D_{x_{3}} \times\left[0, x_{3}\right]\right\}$.

We suppose that there is a positive constant $\gamma_{1}$ such that

$$
\int_{D_{x_{3}}} C_{i j k l} u_{i, j} u_{k, l} \mathrm{~d} a \geq \gamma_{1} \int_{D_{x_{3}}} u_{i, j} u_{i, j} \mathrm{~d} a
$$

and hence Eq. (4.5) gives

$$
-\mathcal{K}_{1}^{\prime}\left(x_{3}\right) \geq \gamma_{1} \int_{D_{x_{3}}} u_{i, j} u_{i, j} \mathrm{~d} a
$$

By means of the Cauchy-Schwarz inequality and the arithmetic-geometric mean inequality, from the relations (3.21), (3.22) and (4.3), we deduce that

$$
\left|\mathcal{K}_{1}\left(x_{3}\right)\right| \leq \frac{M_{1}}{\sqrt{\lambda_{0}}} \int_{D_{x_{3}}} u_{i, j} u_{i, j} \mathrm{~d} a,
$$

where

$$
M_{1}=\left(C_{i 3 k l} C_{i 3 k l}\right)^{1 / 2} .
$$


Therefore, the relations (4.8) and (4.9) lead to the following first-order differential inequality:

$$
\left|\mathcal{K}_{1}\left(x_{3}\right)\right|+\frac{1}{v_{1}} \mathcal{K}_{1}^{\prime}\left(x_{3}\right) \leq 0
$$

where

$$
v_{1}=\frac{\gamma_{1} \sqrt{\lambda_{0}}}{M_{1}} .
$$

Let us consider the case of the finite cylinder. In view of the end boundary condition (2.10) and the relation (4.1), we obtain

$$
\mathcal{K}_{1}(L)=0 .
$$

Then, the relation (4.6) gives

$$
\mathcal{K}_{1}\left(x_{3}\right)=\int_{\Omega_{x_{3}}} C_{i j k l} u_{i, j} u_{k, l} \mathrm{~d} v,
$$

so that, in view of the assumption (4.7), we can conclude that

$$
\mathcal{K}_{1}\left(x_{3}\right) \geq 0 \quad \text { for all } x_{3} \in[0, L] .
$$

Thus, $\mathcal{K}_{1}\left(x_{3}\right)$ appears as an acceptable measure of the displacement.

Moreover, by integrating the differential inequality (4.11), we obtain, for the finite cylinder, the following spatial decay estimate

$$
\mathcal{K}_{1}\left(x_{3}\right) \leq \mathcal{K}_{1}(0) e^{-v_{1} x_{3}} \quad \text { for all } x_{3} \in[0, L]
$$

where $v_{1}$ is given by the relation (4.12).

Let us now study the case of a semi-infinite cylinder (that is the case when $L \rightarrow \infty$ ). In view of the relation (4.6) we can conclude that $\mathcal{K}_{1}(\infty) \equiv \lim _{L \rightarrow \infty} \mathcal{K}_{1}(L)$ exists and is finite if and only if there is finite the energy $\mathcal{E}_{1}\left(x_{3}\right)$ associated with the displacement vector $u_{i}$, that is

$$
\mathcal{E}_{1}\left(x_{3}\right)=\int_{\Omega_{x_{3}}} C_{i j k l} u_{i, j} u_{k, l} \mathrm{~d} v<\infty .
$$

We further recall the relation (4.8) so that $\mathcal{K}_{1}\left(x_{3}\right)$ is a non-increasing function with respect to $x_{3}$ on the interval $[0, \infty)$. Consequently, we distinguish the following cases: (1) $\mathcal{K}_{1}(\infty) \geq 0$ and (2) $\mathcal{K}_{1}(\infty)<0$.

Since $0 \leq \mathcal{K}_{1}(\infty) \leq \mathcal{K}_{1}\left(x_{3}\right)$ for all $x_{3} \in[0, \infty)$, in the case (1) we deduce that $K_{1}\left(x_{3}\right)$ satisfies a decay law of the type described into relation (4.16). In the case (2) we deduce that there exists $x_{3}^{0} \in[0, \infty)$ such that $\mathcal{K}_{1}\left(x_{3}^{0}\right)<0$. Then we conclude that

$$
\mathcal{K}_{1}\left(x_{3}\right)<0 \text { for all } x_{3} \in\left[x_{3}^{0}, \infty\right)
$$

and hence the differential inequality (4.11) gives

$$
\frac{d}{\mathrm{~d} x_{3}}\left[e^{-v_{1} x_{3}} \mathcal{K}_{1}\left(x_{3}\right)\right] \leq 0 \text { for all } x_{3} \in\left[x_{3}^{0}, \infty\right)
$$


which, by an integration, furnishes

$$
-\mathcal{K}_{1}\left(x_{3}\right) \geq-\mathcal{K}_{1}\left(x_{3}^{0}\right) e^{v_{1}\left(x_{3}-x_{3}^{0}\right)} \quad \text { for all } x_{3} \in\left[x_{3}^{0}, \infty\right)
$$

As a direct consequence, from the relation (4.20) we obtain that $\mathcal{K}_{1}(\infty)=-\infty$, a conclusion that proves that the energy $\mathcal{E}_{1}\left(x_{3}\right)$ is infinite.

We may summarize the result obtained in the following theorem.

Theorem 2 Suppose that the hypothesis (4.7) holds true. For the finite cylinder consisting of a monoclinic material, the function $\mathcal{K}_{1}\left(x_{3}\right)$ defined by Eq. (4.3) represents a measure that satisfies the decay law described by the relation (4.16). In the context of the monoclinic semi-infinite cylinder we have

$$
\mathcal{E}_{1}\left(x_{3}\right) \leq \mathcal{E}_{1}(0) e^{-v_{1} x_{3}} \quad \text { for all } x_{3} \in[0, \infty),
$$

provided that the displacement has a finite energy, $\mathcal{E}_{1}(0)<\infty$.

Remark 2 Notice that the hypothesis (4.7) characterizes a class of strongly elliptic elastic materials. It has to be considered as a restriction on the elastic constants in the sense in which we have considered the relations (3.14), (3.16) or (3.51). An inequality of this sort will be considered later for elastic materials with transverse isotropy which will be proved to be a consequence of the strong ellipticity condition.

Remark 3 The estimates (4.16) and (4.21) may be made fully explicit by obtaining an explicit upper bound for $\mathcal{K}_{1}(0)$ or $\mathcal{E}_{1}(0)$ in terms of the given data. This problem can be handled in a number of ways. In what follows we use an idea devised by Flavin [12] in the study of the spatial decay for a generalized biharmonic equation.

Let $v$ and $w$ be any two smooth vector fields having the same boundary values as $u$. In view of the assumption (4.7) we can define the following scalar product

$$
(v, w)=\int_{\Omega} C_{i j k l} v_{i, j} w_{k, l} \mathrm{~d} v
$$

and note that the equilibrium Eq. (2.1) and the boundary conditions (2.8) to (2.10) imply that

$$
(u, v)=(u, u)=\mathcal{E}_{1}(0)=-\int_{D_{0}} C_{i 3 k l} u_{k, l} g_{i} \mathrm{~d} a .
$$

By using the Schwarz inequality, from the relation (4.23) we can establish the following estimate:

$$
\mathcal{E}_{1}(0) \leq \int_{\Omega} C_{i j k l} v_{i, j} v_{k, l} \mathrm{~d} v
$$

where $v$ is a smooth vector field satisfying the same boundary conditions as $u$. 


\subsection{Second Measure}

In this subsection we introduce the following function

$$
\mathcal{K}_{2}\left(x_{3}\right)=-\int_{D_{x_{3}}}\left(C_{i 3 k 3} u_{k, 3}+C_{i \alpha k 3} u_{k, \alpha}\right) u_{i} \mathrm{~d} a, \quad x_{3} \in[0, L],
$$

so that we obtain

$$
\mathcal{K}_{2}^{\prime}\left(x_{3}\right)=-\int_{D_{x_{3}}}\left(C_{i 3 k 3} u_{i, 3} u_{k, 3}+C_{i 3 k 3} u_{i} u_{k, 33}+C_{i \alpha k 3} u_{i, 3} u_{k, \alpha}+C_{i \alpha k 3} u_{i} u_{k, 3 \alpha}\right) \mathrm{d} a .
$$

By using the equilibrium Eq. (3.55) and by an integration by parts with the use of the lateral boundary condition (2.8), we further deduce that

$$
\mathcal{K}_{2}^{\prime}\left(x_{3}\right)=-\int_{D_{x_{3}}} C_{i j k l} u_{i, l} u_{k, j} \mathrm{~d} a .
$$

Now we assume that there exists a positive constant $\gamma_{2}$ such that

$$
\int_{D_{x_{3}}} C_{i j k l} u_{i, l} u_{k, j} \mathrm{~d} a \geq \gamma_{2} \int_{D_{x_{3}}} u_{i, j} u_{i, j} \mathrm{~d} a,
$$

so that the relation (4.27) furnishes

$$
-\mathcal{K}_{2}^{\prime}\left(x_{3}\right) \geq \gamma_{2} \int_{D_{x_{3}}} u_{i, j} u_{i, j} \mathrm{~d} a .
$$

Proceeding as in the above subsection we can see that $\mathcal{K}_{2}\left(x_{3}\right)$ represents a measure satisfying a differential inequality of the same type like $\mathcal{K}_{1}\left(x_{3}\right)$. Thus, for the measure $\mathcal{K}_{2}\left(x_{3}\right)$ we can establish some spatial estimates of the types described by the relations (4.16) and (4.21).

It is evident from the above analysis that one can define other measures of the same types as $\mathcal{K}_{1}$ and $\mathcal{K}_{2}$. Thus, it appears as possible to get information upon the spatial evolution of the displacement in the whole class of strongly elliptic materials by means of a set of measures of the type considered above. Our task is to find a measure (or a couple of measures) that are able to furnish information on the spatial evolution of the displacement for the entire class of anisotropic and strongly elliptic elastic materials. However, this task can be too complex for general anisotropic elastic materials. One of the reasons for this is that, actually, we do not know explicit necessary and sufficient conditions for the strong ellipticity to hold in anisotropic materials other than those with transverse isotropy. We proceed to pursue such a direction in the next subsection for a particularly important class of anisotropic elastic materials, namely that of transversely isotropic solids.

\subsection{Transversely Isotropic Materials}

Many natural and man-made materials are classified as transversely isotropic (or hexagonal). Such materials are characterized by the fact that one can find a line that allows a rotation of the material about it without changing its properties. The plane, which is perpendicular to this line (the axis of rotational symmetry) is called a plane 
of elastic symmetry or plane of isotropy. A modern example for such a material is a laminate made of randomly oriented chopped fibers that are in general placed in a certain plane. The effective material properties for a bundled structure have no profound direction in that plane, which then becomes a plane of elastic symmetry. Hence, each plane that contains the axis of rotation is a plane of symmetry, and therefore, a transversely isotropic material admits an infinite number of elastic symmetries.

Necessary and sufficient conditions for strong ellipticity to hold for a transversely isotropic linearly elastic solid are established by Merodio and Ogden [13]. In this connection we recall the standard notation

$$
\begin{aligned}
& c_{i j}=C_{i i j j}, \quad i, j \in\{1,2,3\}, \quad(\text { not summed }), \quad c_{22}=c_{11}, \quad c_{23}=c_{13}, \\
& c_{44}=c_{55}=C_{2323}=C_{1313}, \quad c_{66}=C_{1212}=\frac{1}{2}\left(c_{11}-c_{12}\right),
\end{aligned}
$$

corresponding to the direction of transverse isotropy coinciding with the $x_{3}$ coordinate axis. Apart from terms obtained by use of the symmetries Eq. (2.2), these are the only non-zero components of $C_{i j k l}$. Then the necessary and sufficient conditions for strong ellipticity to hold are [13]

$$
c_{11}>0, \quad c_{33}>0, \quad c_{55}>0, \quad c_{11}>c_{12},
$$

$$
\left|c_{13}+c_{55}\right|<c_{55}+\sqrt{c_{11} c_{33}}
$$

Now we note that the relations (3.52) and (3.57) give

$$
\begin{aligned}
\frac{d^{2}}{\mathrm{~d} x_{3}^{2}} \int_{D_{x_{3}}} & {\left[c_{55}\left(u_{1}^{2}+u_{2}^{2}\right)+c_{33} u_{3}^{2}\right] \mathrm{d} a=2 \int_{D_{x_{3}}}\left[c_{11}\left(u_{1,1}^{2}+u_{2,2}^{2}\right)+2 c_{12} u_{1,1} u_{2,2}+\right.} \\
& \left.+\left(c_{13}+c_{55}\right)\left(u_{1,1}+u_{2,2}\right) u_{3,3}+c_{33} u_{3,3}^{2}+c_{66}\left(u_{1,2}^{2}+u_{2,1}^{2}+2 u_{1,2} u_{2,1}\right)\right] \mathrm{d} a+ \\
& +2 \int_{D_{x_{3}}}\left[c_{55}\left(u_{1,3}^{2}+u_{3,1}^{2}+u_{2,3}^{2}+u_{3,2}^{2}\right)+\left(c_{13}+c_{55}\right)\left(u_{1,3} u_{3,1}+u_{2,3} u_{3,2}\right)\right] \mathrm{d} a .
\end{aligned}
$$

Furthermore, we use integration by parts and the lateral boundary condition (2.8) in order to establish the following identity:

$$
\int_{D_{x_{3}}} u_{\alpha, \alpha} u_{3,3} \mathrm{~d} a=\left(\int_{D_{x_{3}}} u_{\alpha, \alpha} u_{3} \mathrm{~d} a\right)_{, 3}+\int_{D_{x_{3}}} u_{\alpha, 3} u_{3, \alpha} \mathrm{d} a .
$$


By combining the identities (4.33) and (4.34) we can generate identities of the following type:

$$
\begin{gathered}
\frac{d^{2}}{\mathrm{~d} x_{3}^{2}}\left\{\int_{D_{x_{3}}}\left[c_{55}\left(u_{1}^{2}+u_{2}^{2}\right)+c_{33} u_{3}^{2}\right] \mathrm{d} a+2 \int_{\Omega_{x_{3}}}\left(c_{55}-c_{13}-2 \kappa\right) u_{\alpha, \alpha} u_{3} \mathrm{~d} v\right\}= \\
=2 \int_{D_{x_{3}}}\left[c_{11}\left(u_{1,1}^{2}+u_{2,2}^{2}\right)+2 c_{12} u_{1,1} u_{2,2}\right. \\
\left.+2\left(c_{13}+\kappa\right) u_{\alpha, \alpha} u_{3,3}+c_{33} u_{3,3}^{2}+c_{66}\left(u_{1,2}+u_{2,1}\right)^{2}\right] \mathrm{d} a \\
+2 \int_{D_{x_{3}}}\left[c_{55}\left(u_{1,3}^{2}+u_{3,1}^{2}+u_{2,3}^{2}+u_{3,2}^{2}\right)+\right. \\
\left.+2\left(c_{55}-\kappa\right)\left(u_{1,3} u_{3,1}+u_{2,3} u_{3,2}\right)\right] \mathrm{d} a,
\end{gathered}
$$

with $\kappa$ a parameter at our disposal. Thus, we can introduce the following measure:

$$
\mathcal{K}_{\kappa}\left(x_{3}\right)=-\int_{D_{x_{3}}}\left\{c_{55} u_{\alpha} u_{\alpha, 3}+\left[c_{33} u_{3,3}-\left(c_{55}-c_{13}-2 \kappa\right) u_{\alpha, \alpha}\right] u_{3}\right\} \mathrm{d} a,
$$

so that the relation (4.35) becomes

$$
\begin{aligned}
\mathcal{K}_{\kappa}^{\prime}\left(x_{3}\right)=- & \int_{D_{x_{3}}}\left[c_{11}\left(u_{1,1}+u_{2,2}\right)^{2}+2\left(c_{13}+\kappa\right)\left(u_{1,1}+u_{2,2}\right) u_{3,3}+c_{33} u_{3,3}^{2}+\right. \\
& \left.+c_{66}\left(u_{1,2}-u_{2,1}\right)^{2}\right] \mathrm{d} a \\
& -\int_{D_{x_{3}}}\left[c_{55}\left(u_{1,3}^{2}+u_{3,1}^{2}+u_{2,3}^{2}+u_{3,2}^{2}\right)+2\left(c_{55}-\kappa\right)\left(u_{1,3} u_{3,1}+u_{2,3} u_{3,2}\right)\right] \mathrm{d} a .
\end{aligned}
$$

We note that the relation (4.32) can be written in the equivalent form

$$
-2 c_{55}-\sqrt{c_{11} c_{33}}<c_{13}<\sqrt{c_{11} c_{33}} \text {. }
$$

Moreover, for any $c_{13}$ which satisfies the inequality Eq. (4.38), it is possible to determine $\kappa \in\left(0,2 c_{55}\right)$ so that

$$
\left|\kappa+c_{13}\right|<\sqrt{c_{11} c_{33}} .
$$

In fact, in view of the relation (4.38), the choice for $\kappa$ must be such that

$$
\max \left(-c_{13}-\sqrt{c_{11} c_{33}}, 0\right)<\kappa<\min \left(2 c_{55},-c_{13}+\sqrt{c_{11} c_{33}}\right) .
$$

Throughout in the remainder of this section we will assume that the arbitrary parameter $\kappa$ satisfies the relation (4.40). 
With this choice, the relation (4.39) implies that

$$
\begin{gathered}
\int_{D_{x_{3}}}\left[c_{11}\left(u_{1,1}+u_{2,2}\right)^{2}+2\left(c_{13}+\kappa\right)\left(u_{1,1}+u_{2,2}\right) u_{3,3}+c_{33} u_{3,3}^{2}\right] \mathrm{d} a \geq \\
\geq \omega_{1} \int_{D_{x_{3}}}\left[\left(u_{1,1}+u_{2,2}\right)^{2}+u_{3,3}^{2}\right] \mathrm{d} a,
\end{gathered}
$$

where

$$
\omega_{1}=\frac{1}{2}\left[c_{11}+c_{33}-\sqrt{\left(c_{11}-c_{33}\right)^{2}+4\left(c_{13}+\kappa\right)^{2}}\right] .
$$

By using the same choice, we deduce that

$$
\left|c_{55}-\kappa\right|<c_{55}
$$

and hence we obtain that

$$
\begin{gathered}
\int_{D_{x_{3}}}\left[c_{55}\left(u_{1,3}^{2}+u_{3,1}^{2}+u_{2,3}^{2}+u_{3,2}^{2}\right)+2\left(c_{55}-\kappa\right)\left(u_{1,3} u_{3,1}+u_{2,3} u_{3,2}\right)\right] \mathrm{d} a \geq \\
\geq \omega_{2} \int_{D_{x_{3}}}\left(u_{1,3}^{2}+u_{3,1}^{2}+u_{2,3}^{2}+u_{3,2}^{2}\right) \mathrm{d} a
\end{gathered}
$$

where

$$
\omega_{2}=\min \left(\kappa, 2 c_{55}-\kappa\right) .
$$

The estimates (4.41) and (4.44) when substituted into relation (4.37) give

$$
\begin{gathered}
-\mathcal{K}_{\kappa}^{\prime}\left(x_{3}\right) \geq m_{\kappa} \int_{D_{x_{3}}} u_{\alpha, \beta} u_{\alpha, \beta} \mathrm{d} a+\omega_{2} \int_{D_{x_{3}}}\left(u_{3, \alpha} u_{3, \alpha}+u_{\alpha, 3} u_{\alpha, 3}\right) \mathrm{d} a+ \\
+\omega_{1} \int_{D_{x_{3}}} u_{3,3}^{2} \mathrm{~d} a, \quad \text { for all } x_{3} \in[0, L],
\end{gathered}
$$

where

$$
m_{\kappa}=\min \left(\omega_{1}, c_{66}\right)
$$

On the other hand, by means of the Schwarz inequality and the arithmeticgeometric mean inequality and the relations (3.21) and (3.22), from the relation (4.36) we obtain

$$
\begin{aligned}
\left|\mathcal{K}_{\kappa}\left(x_{3}\right)\right| \leq & \frac{1}{2 \sqrt{\lambda_{0}}}\left(c_{55}+\left|c_{55}-c_{13}-2 \kappa\right|\right) \int_{D_{x_{3}}} u_{\alpha, \beta} u_{\alpha, \beta} \mathrm{d} a+ \\
& +\frac{1}{2 \sqrt{\lambda_{0}}}\left(c_{33}+\left|c_{55}-c_{13}-2 \kappa\right|\right) \int_{D_{x_{3}}} u_{3, \alpha} u_{3, \alpha} \mathrm{d} a+ \\
& +\frac{c_{55}}{2 \sqrt{\lambda_{0}}} \int_{D_{x_{3}}} u_{\alpha, 3} u_{\alpha, 3} \mathrm{~d} a+\frac{c_{33}}{2 \sqrt{\lambda_{0}}} \int_{D_{x_{3}}} u_{3,3}^{2} \mathrm{~d} a .
\end{aligned}
$$


Therefore, the relations (4.46) and (4.48) furnish the following first-order differential inequality:

$$
\left|\mathcal{K}_{\kappa}\left(x_{3}\right)\right|+\frac{1}{v_{\kappa}} \mathcal{K}_{\kappa}^{\prime}\left(x_{3}\right) \leq 0 \quad \text { for all } x_{3} \in[0, L],
$$

where

$$
\frac{1}{v_{\kappa}}=\frac{1}{2 \sqrt{\lambda_{0}}} \max \left[\frac{c_{33}}{\omega_{1}}, \frac{c_{55}}{\omega_{2}}, \frac{1}{m_{\kappa}}\left(c_{55}+\left|c_{55}-c_{13}-2 \kappa\right|\right), \frac{1}{\omega_{2}}\left(c_{33}+\left|c_{55}-c_{13}-2 \kappa\right|\right)\right] \text {. }
$$

We summarize the result obtained in the following theorem.

Theorem 3 Suppose that the cylinder consists of a transversely isotropic elastic material. Then the function $\mathcal{K}_{\kappa}\left(x_{3}\right)$ defined by Eq. (4.36) represents a measure that satisfies a decay law of type described by the relation (4.16). In the context of the monoclinic semi-infinite cylinder we have

$$
\mathcal{E}_{\kappa}\left(x_{3}\right) \leq \mathcal{E}_{\kappa}(0) e^{-v_{\kappa} x_{3}} \quad \text { for all } x_{3} \in[0, \infty),
$$

provided that the displacement has a finite energy, $\mathcal{E}_{\kappa}(0)<\infty$, where

$$
\begin{aligned}
\mathcal{E}_{\kappa}\left(x_{3}\right)= & \int_{\Omega_{x_{3}}}\left[c_{11}\left(u_{1,1}+u_{2,2}\right)^{2}+2\left(c_{13}+\kappa\right)\left(u_{1,1}+u_{2,2}\right) u_{3,3}+c_{33} u_{3,3}^{2}+\right. \\
& \left.+c_{66}\left(u_{1,2}-u_{2,1}\right)^{2}\right] d v \\
& +\int_{\Omega_{x_{3}}}\left[c_{55}\left(u_{1,3}^{2}+u_{3,1}^{2}+u_{2,3}^{2}+u_{3,2}^{2}\right)+2\left(c_{55}-\kappa\right)\left(u_{1,3} u_{3,1}+u_{2,3} u_{3,2}\right)\right] d v
\end{aligned}
$$

Remark 4 The estimate (4.51) may be made fully explicit by obtaining an explicit upper bound for $\mathcal{K}_{\kappa}(0)$ or $\mathcal{E}_{\kappa}(0)$ in terms of the given data. This problem can be handled as in the Subsection 4.1.

Remark 5 The identity Eq. (4.33) allows us to consider the function

$$
K_{\kappa}\left(x_{3}\right)=\int_{D_{x_{3}}}\left(c_{55} u_{\alpha} u_{\alpha}+c_{33} u_{3}^{2}\right) \mathrm{d} a+2 \int_{\Omega_{x_{3}}}\left(c_{55}-c_{13}-2 \kappa\right) u_{\alpha, \alpha} u_{3} \mathrm{~d} v
$$

as a possible alternative measure for discussing the spatial behaviour of the displacement. It is related to $\mathcal{K}_{\kappa}\left(x_{3}\right)$ by

$$
K_{\kappa}^{\prime}\left(x_{3}\right)=-2 \mathcal{K}_{\kappa}\left(x_{3}\right) .
$$

Obviously, for the semi-infinite cylinder (i.e., when $L \rightarrow \infty$, say) we have to assume that the volume integral in Eq. (4.53) is convergent. In such case, it follows that

$$
\lim _{x_{3} \rightarrow \infty} \int_{\Omega_{x_{3}}}\left(c_{55}-c_{13}-2 \kappa\right) u_{\alpha, \alpha} u_{3} \mathrm{~d} v=0
$$


and hence

$$
\lim _{x_{3} \rightarrow \infty} K_{\kappa}\left(x_{3}\right)=\lim _{x_{3} \rightarrow \infty} \int_{D_{x_{3}}}\left(c_{55} u_{\alpha} u_{\alpha}+c_{33} u_{3}^{2}\right) \mathrm{d} a
$$

that is, $K_{\kappa}\left(x_{3}\right)$ behaves at infinity like the measure $I\left(x_{3}\right)$ or $J\left(x_{3}\right)$. Thus, $K_{\kappa}\left(x_{3}\right) \rightarrow 0$ as $x_{3} \rightarrow \infty$ implies that $u_{i} \rightarrow 0$ as $x_{3} \rightarrow \infty$.

For convenience, in what follows we will consider the case of a semi-infinite cylinder and we will assume that the displacement has a finite energy, $\mathcal{E}_{\kappa}(0)<\infty$, so that we have a spatial decay as described by the relation (4.51).

In view of the relations (4.37) and (4.54), we have

$$
\begin{gathered}
K_{\kappa}^{\prime \prime}\left(x_{3}\right)=2 \int_{D_{x_{3}}}\left[c_{11}\left(u_{1,1}+u_{2,2}\right)^{2}+2\left(c_{13}+\kappa\right)\left(u_{1,1}+u_{2,2}\right) u_{3,3}+\right. \\
\left.+c_{33} u_{3,3}^{2}+c_{66}\left(u_{1,2}-u_{2,1}\right)^{2}\right] \mathrm{d} a+ \\
+2 \int_{D_{x_{3}}}\left[c_{55}\left(u_{1,3}^{2}+u_{3,1}^{2}+u_{2,3}^{2}+u_{3,2}^{2}\right)+\right. \\
\left.+2\left(c_{55}-\kappa\right)\left(u_{1,3} u_{3,1}+u_{2,3} u_{3,2}\right)\right] \mathrm{d} a .
\end{gathered}
$$

Moreover, on the basis of the relations (4.46) and (4.54), we obtain

$$
K_{\kappa}^{\prime}\left(x_{3}\right)=-2 \mathcal{E}_{\kappa}\left(x_{3}\right),
$$

and

$$
\begin{aligned}
K_{\kappa}^{\prime \prime}\left(x_{3}\right) \geq & 2 m_{\kappa} \int_{D_{x_{3}}} u_{\alpha, \beta} u_{\alpha, \beta} \mathrm{d} a+2 \omega_{2} \int_{D_{x_{3}}}\left(u_{3, \alpha} u_{3, \alpha}+u_{\alpha, 3} u_{\alpha, 3}\right) \mathrm{d} a+ \\
& +2 \omega_{1} \int_{D_{x_{3}}} u_{3,3}^{2} \mathrm{~d} a, \quad \text { for all } x_{3} \in[0, L], \\
-K_{\kappa}^{\prime}\left(x_{3}\right) \geq & 2 m_{\kappa} \int_{\Omega_{x_{3}}} u_{\alpha, \beta} u_{\alpha, \beta} \mathrm{d} a+2 \omega_{2} \int_{\Omega_{x_{3}}}\left(u_{3, \alpha} u_{3, \alpha}+u_{\alpha, 3} u_{\alpha, 3}\right) \mathrm{d} a+ \\
& +2 \omega_{1} \int_{\Omega_{x_{3}}} u_{3,3}^{2} \mathrm{~d} a, \quad \text { for all } x_{3} \in[0, L] .
\end{aligned}
$$

On the other hand, by means of the Schwarz inequality and the arithmeticgeometric mean inequality and the relations (3.21) and (3.22), from the relation (4.53) we find that

$$
\begin{gathered}
K_{\kappa}\left(x_{3}\right) \leq \frac{c_{55}}{\lambda_{0}} \int_{D_{x_{3}}} u_{\alpha, \beta} u_{\alpha, \beta} \mathrm{d} a+\frac{c_{33}}{\lambda_{0}} \int_{D_{x_{3}}} u_{3, \alpha} u_{3, \alpha} \mathrm{d} a+ \\
+\frac{\left|c_{55}-c_{13}-2 \kappa\right|}{\sqrt{\lambda_{0}}} \int_{\Omega_{x_{3}}}\left(u_{\alpha, \beta} u_{\alpha, \beta}+u_{3, \alpha} u_{3, \alpha}\right) \mathrm{d} v .
\end{gathered}
$$


Therefore, from the relations (4.59) to (4.61) we deduce the following secondorder differential inequality,

$$
K_{\kappa}^{\prime \prime}\left(x_{3}\right)-a K_{\kappa}^{\prime}\left(x_{3}\right)-b K_{\kappa}\left(x_{3}\right) \geq 0 \quad \text { for all } x_{3} \in[0, L],
$$

where

$$
a=\frac{\Lambda_{2}}{\Lambda_{1}}, \quad b=\frac{1}{\Lambda_{1}}
$$

with

$$
\Lambda_{1}=\frac{1}{2 \lambda_{0}} \max \left(\frac{c_{55}}{m_{\kappa}}, \frac{c_{33}}{\omega_{2}}\right), \quad \Lambda_{2}=\frac{\left|c_{55}-c_{13}-2 \kappa\right|}{2 \sqrt{\lambda_{0}}} \max \left(\frac{1}{m_{\kappa}}, \frac{1}{\omega_{2}}\right) .
$$

By the Comparison Principle, from Eq. (4.62) it follows that $K_{\kappa}\left(x_{3}\right)$ is bounded above by $G\left(x_{3}\right)$, the solution of the differential equation

$$
G^{\prime \prime}\left(x_{3}\right)-a G^{\prime}\left(x_{3}\right)-b G\left(x_{3}\right)=0, \quad \text { for all } x_{3} \in[0, L],
$$

with the boundary conditions

$$
G(0)=K_{\kappa}(0), \quad G(L)=K_{\kappa}(L) .
$$

On this basis, we can write

$$
\begin{aligned}
K_{\kappa}\left(x_{3}\right) & \leq \frac{1-e^{-\left(\sigma_{1}+\sigma_{2}\right)\left(L-x_{3}\right)}}{1-e^{-\left(\sigma_{1}+\sigma_{2}\right) L}} K_{\kappa}(0) e^{-\sigma_{2} x_{3}}+\frac{1-e^{-\left(\sigma_{1}+\sigma_{2}\right) x_{3}}}{1-e^{-\left(\sigma_{1}+\sigma_{2}\right) L}} K_{\kappa}(L) e^{-\sigma_{1}\left(L-x_{3}\right)} \leq \\
& \leq K_{\kappa}(0) e^{-\sigma_{2} x_{3}}+K_{\kappa}(L) e^{-\sigma_{1}\left(L-x_{3}\right)},
\end{aligned}
$$

where

$$
\sigma_{1}=\frac{1}{2}\left(a+\sqrt{a^{2}+4 b}\right), \quad \sigma_{2}=\frac{1}{2}\left(-a+\sqrt{a^{2}+4 b}\right) .
$$

This result is embodied in the following theorem.

\section{Theorem 4 If}

$$
\lim _{L \rightarrow \infty} K_{\kappa}(L) e^{-\sigma_{1} L}=0
$$

then

$$
K_{\kappa}\left(x_{3}\right) \leq K_{\kappa}(0) e^{-\sigma_{2} x_{3}} \quad \text { for all } x_{3} \in[0, \infty)
$$

Remark 6 From the relations (2.9) and (4.53) we deduce that

$$
K_{\kappa}(0)=\int_{D_{0}}\left(c_{55} g_{\alpha} g_{\alpha}+c_{33} g_{3}^{2}\right) \mathrm{d} a+2 \int_{\Omega}\left(c_{55}-c_{13}-2 \kappa\right) u_{\alpha, \alpha} u_{3} \mathrm{~d} v .
$$

So, in order to make fully explicit the estimate (4.70), we note that the second integral in Eq. (4.71) can be bounded above by $\mathcal{E}_{\kappa}(0)$, which further can be estimated in terms of the given data. 
Figure 2 Dependence of the decay rates $v_{\kappa}$ and $\sigma_{2}$ on the parameter $\kappa$.

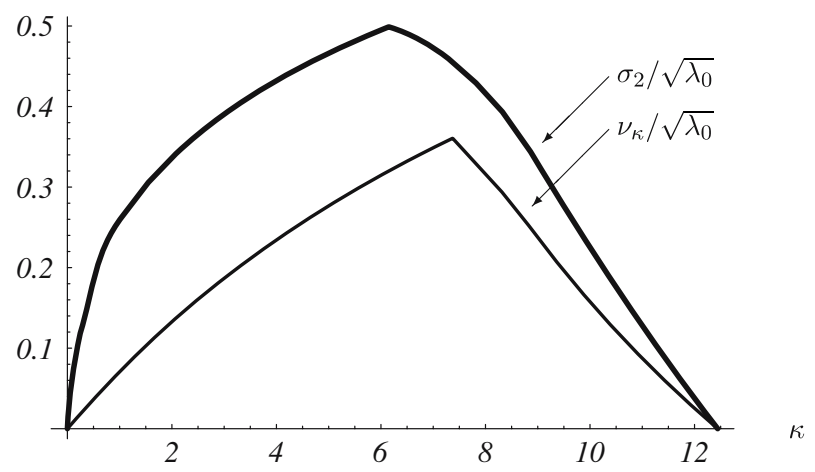

Remark 7 It follows from the relations (4.51) and (4.58) that $K_{\kappa}\left(x_{3}\right)$ is an acceptable measure of the displacement because we have

$$
K_{\kappa}\left(x_{3}\right)=2 \int_{x_{3}}^{\infty} \mathcal{E}_{\kappa}(\eta) \mathrm{d} \eta
$$

Remark 8 It is possible that the use of $K_{\kappa}\left(x_{3}\right)$ can predict a more accurate decay rate than that obtained by the use of $\mathcal{K}_{\kappa}\left(x_{3}\right)$, as the following particular example shows.

The dependence of the decay rates $v_{\kappa}$ and $\sigma_{2}$ on the parameter $\kappa$ is illustrated in the Figure 2, where the transversely isotropic elastic constants used are those determined by Yoon et al. [14, relation (33)] starting with the set of orthotropic elastic constants for cortical bone measured by Ashman et al. [15]. That means we have considered the values: $c_{11}=c_{22}=19.026, c_{33}=27.524, c_{12}=9.957, c_{13}=c_{23}=10.441, c_{44}=c_{55}=$ $11.824, c_{66}=9.069$, in the dimension of GPa. In Figure 2 it is also obvious that, in order to have a rapid decay we must take $\kappa \approx 6.149$. Using this last value we obtain the spatial decay of the measures $\mathcal{K}_{\kappa}$ and $K_{\kappa}$ with respect to $x_{3}$ as it is illustrated in Figure 3, for the same material.

Figure 3 Spatial decay of $\mathcal{K}_{\kappa}$ and $K_{\kappa}$ with respect to $x_{3}$.

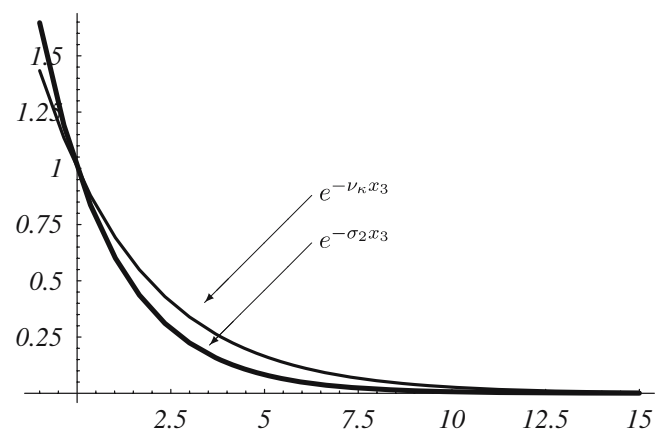




\section{Concluding Comments}

The spatial behaviour in the constrained elastic cylinder of variable cross-section made by an isotropic and homogeneous linear elastic material is studied in [1]. The present purpose is to extend some of these studies to include a whole class of anisotropic elastic materials under the assumption of strong ellipticity, instead of the positive definiteness of the elasticity tensor. It is believed that the mean-square cross-sectional measures $I\left(x_{3}\right)$ and $J\left(x_{3}\right)$ as defined by the relations (3.6) and (3.52), respectively, or those obtained from them by particularizing to various classes of symmetric systems, can be used to obtain spatial evolution of the displacement for important classes of anisotropic materials.

On the other hand, measures containing the displacement and its first gradient corresponding to the functions of type $\mathcal{K}_{1}, \mathcal{K}_{2}$ are intended to be used in order to obtain information upon the spatial behaviour under the strong ellipticity condition. In the present paper we have used the results by Merodio and Ogden [13], concerning the strong ellipticity for transversely isotropic materials, in order to specify measures able to furnish information on the spatial behaviour of the displacement in the whole class of strongly elliptic transversely isotropic materials. The situation appears to be much more complicated for less symmetric materials where characterization of the strong ellipticity is not yet explicitly known.

Acknowledgements The authors are very grateful to the reviewers for their suggestions and observations which led to an essential improvement of the paper.

\section{References}

1. Flavin, J.N., Knops, R.J., Payne, L.E.: Decay estimates for the constrained elastic cylinder of variable cross section. Q. Appl. Math. 67(2), 325-350 (1989)

2. Biollay, Y.: First boundary value problem in elasticity: Bounds for the displacements and SaintVenant's principle. Z. Angew. Math. Phys. 31, 556-567 (1980)

3. Oleinik, O.A., Yosifian, G.A.: On singularities at the boundary points and uniqueness theorems for solutions of the first boundary value problem of elasticity. Commun. Partial Differ. Equ. 1, 937-969 (1977)

4. Oleinik, O.A., Yosifian, G.A.: Saint-Venant's principle for the mixed boundary value problem of the theory of elasticity and its applications. Dokl. Akad. Nauk SSSR 233, 824-827 (1977) [Soviet. Math. Dokl. 22, 233-235 (1977)]

5. Horgan, C.O., Knowles, J.K.: Recent developments concerning Saint-Venant's principle. In: Wu, T.Y., Hutchinson, J.W. (eds.) Advances Applied Mechanics, vol. 23, pp. 179-269. Academic, New York (1983)

6. Horgan, C.O.: Recent developments concerning Saint-Venant's principle: An update. Appl. Mech. Rev. 42(11), 295-303 (1989)

7. Horgan, C.O.: Recent developments concerning Saint-Venant's principle: A second update. Appl. Mech. Rev. 49(10), S101-S111 (1996)

8. Horgan, C.O., Carlsson, L.A.: Saint-Venant end effects for anisotropic materials. In: Kelly, A., Zweben, C. (eds.) Comprehensive Composite Materials, vol. 5, pp. 5-21. Elsevier, Oxford, UK (2000)

9. Gurtin, M.E.: The linear theory of elasticity. In: Truesdell, C. (ed.) Handbuch der Physik, vol.VIa/2. Springer, Berlin Heidelberg New York (1972)

10. Fichera, G.: Existence theorems in elasticity. In: Truesdell, C. (ed.) Handbuch der Physik, vol.VIa/2. Springer, Berlin Heidelberg New York (1972)

11. Flavin, J.N., Rionero, S.: Qualitative Estimates for Partial Differential Equations: An Introduction. CRC, Boca Raton, Florida (1995)

Springer 
12. Flavin, J.N.: Spatial-decay estimates for a generalized biharmonic equation in inhomogeneous elasticity. J. Eng. Math. 46, 241-252 (2003)

13. Merodio, J., Ogden, R.W.: A note on strong ellipticity for transversely isotropic linearly elastic solids. Q. J. Mech. Appl. Math. 56(4), 589-591 (2003)

14. Yoon, Y.J., Yang, G., Cowin, S.C.: Estimation of the effective transversely isotropic elastic constants of a material from known values of the material's orthotropic elastic constants. Biomechan. Model Mechanobiol. 1, 83-93 (2002)

15. Ashman, R.B., Cowin, S.C., van Buskirk, W.C., Rice, J.C.: A continuous wave technique for the measurement of the elastic properties of cortical bone. J. Biomech. 17, 349-361 (1984) 